\title{
Computer use and ulnar neuropathy: results from a case-referent study
}

\author{
Andersen $\mathrm{JH}^{\mathrm{a}^{*}}$, Frost $\mathrm{P}^{\mathrm{b}}$, Fuglsang-Frederiksen $\mathrm{A}^{\mathrm{c}}$, Johnson $\mathrm{B}^{\mathrm{c}}$, and Wulff Svendsen $\mathrm{S}^{\mathrm{a}}$ \\ a Danish Ramazzini Centre, Department of Occupational Medicine, Herning Hospital, Gl. Landevej 61, 7400 \\ Herning, Denmark \\ ${ }^{b}$ Danish Ramazzini Centre ,Department of Occupational Medicine, Aarhus Hospital, Aarhus University Hospital, \\ Norrebrogade, 8000 Aarhus C, Denmark \\ c Department of Neurophysiology, Aarhus Hospital, Aarhus University Hospital, Norrebrogade, 8000 Aarhus C, \\ Denmark
}

\begin{abstract}
We aimed to evaluate associations between vocational computer use and 1) ulnar neuropathy, and 2) ulnar neuropathy-like symptoms as distinguished by electroneurography. We identified all patients aged 18-65 years, examined at the Department of Neurophysiology on suspicion of ulnar neuropathy, 2001-2007. We mailed a questionnaire to 546 patients with ulnar neuropathy, 633 patients with ulnar neuropathy-like symptoms, and three community referents per case, matched on sex, age, and primary care centre. From a Job Exposure Matrix we extracted estimates of daily hours of computer use. The analysis was performed by conditional logistic regression.

There were a negative association between daily hours of computer use and the two outcomes of interest. Participants who reported their elbow to be in contact with their working table for 2 hours or more during the workday had an elevated risk for ulnar neuropathy $(\mathrm{OR}=2.16,95 \% \mathrm{CI} ; 1.06-4.44)$.

The two outcomes were not associated with daily hours of computer use. Findings suggested specific effects of pressure on the elbow, and might be an explanation for the overweight of left-sided outcomes in this primarily right-handed group. Preventive efforts would then be straightforward by providing appropriate arm support for the left arm and elbow.
\end{abstract}

Keywords: Entrapment, VDU-use, electroneurography, elbow, job exposure matrix

\section{Introduction}

After carpal tunnel syndrome (CTS), ulnar neuropathy at the elbow is the most common form of nerve entrapment. ${ }^{1}$ Ulnar neuropathy may be diagnosed on the basis of symptoms and signs $[5,8]$, and the clinical diagnosis may be confirmed by electroneurography (ENG). However, the diagnostic weight of ENG is a matter of discussion [5]. For clinically diagnosed ulnar neuropathy at the elbow, a prevalence of $0.6-0.8 \%$ has been found in a French working population [7]. Incidence rates of 20 to 25 per 100,000 person-years have been reported for general population samples in the UK and Italy $[3,4]$.

The aim of the present study was to evaluate if hours of daily use of computers was associated with ulnar neuropathy and ulnar neuropathy-like symptoms as distinguished by ENG, and furthermore to evaluate if leaning on the elbow was a risk factor for ulnar neuropathy and ulnar neuropathy-like symptoms. Other occupational and individual risk factors will be reported in separate papers.

\section{Methods}

\subsection{Design and study population}

We chose a case-referent design as the most efficient way to study exposure-response relations, given the low incidence of ulnar neuropathy. From the Danish National Patient Register we received data on all contacts to the Department of Neurophysiology, Aarhus University Hospital, 1 March 2001 to 30 June 2007 , with a referral diagnosis of mononeuropathy of upper limb (group G56 in the International Classification of Diseases $10^{\text {th }}$ revision (ICD-10)) and a discharge diagnosis of either ulnar neuropathy (ICD-10 
code G56.2) or no neuropathy (ICD-10 codes Z). Data was delivered on 21 August 2007. We extracted patients who were $\geq 18-<65$ years old at the date of their first contact, the index date. For the index dates, we briefly examined neurophysiologic records to identify patients who were examined by ENG across the elbow, which indicates a clinical suspicion of ulnar neuropathy. We excluded patients if they had a traumatic lesion as suggested by very low or absent motor responses. Remaining patients were divided into two case groups on the basis of their discharge diagnosis: 1) cases with ulnar neuropathy, and 2) cases with ulnar neuropathy-like symptoms.

In the Danish National Health Insurance Service Registry we identified all patients who had the same primary health care provider at the index date. After exclusion due to death, emigration, and name and/or address protection, referents were drawn from these base populations, individually matched on sex, age (+/- $2 \frac{1}{2}$ years), and primary health care provider at the index date. Four referents were drawn for each case with ulnar neuropathy, and three for each case with ulnar neuropathy-like symptoms. We conducted a double case-referent study: 1) Cases with ulnar neuropathy versus their matched referents.2) Cases with ulnar neuropathy-like symptoms versus their matched referents.

The study was authorized by the Danish Data Protection Agency. The five Danish administrative regions approved the use of data from the Danish National Health Insurance Service Registry, and the Danish National Board of Health permitted the use of neurophysiologic data. In Denmark, register and questionnaire based studies do not require approval by ethical and scientific committees, nor informed consent.

\subsection{Neurophysiologic examinations and quality as- surance of case status}

ENG was performed with needle or surface electrodes depending on available staff resources and patient preferences. The needle electrode technique included placement of three needle electrodes near the ulnar nerve at the wrist, below the elbow five $\mathrm{cm}$ distal to the medial epicondyle, and above the elbow five $\mathrm{cm}$ proximal to the medial epicondyle. Normal mean values [corrected for age and height] were used. Distal latencies, motor and sensory amplitudes and conduction velocities were expressed in Z-scores, i.e. deviation from normal mean values expressed in standard deviations (SD). According to consensus criteria, definite and/or partial motor conduction block was identified if an amplitude $>0.5 \mathrm{mV}$ of the compound motor action potential was registered from stimulation below the elbow [6].

\subsection{Assessment of occupational biomechanical expo- sures}

We constructed a Job Exposure Matrix (JEM) based on experts' ratings. Exposure estimates for each participant were obtained by combining self-reported job histories with quantitative job exposures from the JEM. Questionnaire data was collected from 7 January to 30 April 2008 with a maximum of two reminders. The questionnaire was individualized so that we asked for the main job title in the year before the index year (index year $=$ year that included the index date). We also asked for up to five preceding job titles and corresponding time periods. Each job title was recoded into one of 2227 occupational titles in the Danish version of the International Standard Classification of Occupations (D-ISCO 88). A total of 806 occupational titles were represented in the questionnaires. We divided these occupational titles into 169 groups of jobs that were expected to have similar exposure profiles with respect to all exposures under study. We then selected 15 job groups that covered a wide range of exposures. Five experts, who were experienced occupational health physicians, consensus rated these 15 job groups so that they could serve as benchmarks during the remaining rating process. In this way the experts could calibrate their estimates to a common scale [9]. For each of the remaining 154 job groups, the experts independently rated the mean number of hours (in half-hours interval) with computer work. For other analysis mean force, repetition, non neutral postures and exposure for hand arm vibrations (HAV-time) were also rated by the experts. Each participant received estimates of daily exposures in the year before the index year.

\subsection{Statistical methods}

We applied conditional logistic regression to analyze risk of each outcome in relation to categorized computer use in times per day, yielding odds ratios for each exposure category with mutual adjustment for a predetermined set of potential confounders, which consisted of physical risk factors from the expert rating: force, repetition, non neutral postures and exposure for hand arm vibrations, and individual factors. A variable for handedness was included in the regression models. 
Table 1

Risk of ulnar neuropathy (study 1) and ulnar neuropathy-like symptoms (study 2 ) in relation to computer work characteristics. In each study, cases and referents were individually matched on sex, age $\left(+/-2 \frac{1}{2}\right.$ years $)$, and primary health care provider.

\begin{tabular}{|c|c|c|c|c|}
\hline & \multicolumn{2}{|c|}{ Study 1: ulnar neuropathy } & \multicolumn{2}{|c|}{ Study 2: Ulnar neuropathy-like symptoms } \\
\hline Computer work & Unadjusted OR & Fully adjusted OR* & Unadjusted OR & Fully adjusted $\mathrm{OR}^{*}$ \\
\hline characteristics & $(95 \% \mathrm{CI})$ & $(95 \% \mathrm{CI})$ & $(95 \% \mathrm{CI})$ & $(95 \% \mathrm{CI})$ \\
\hline \multicolumn{5}{|l|}{ Hours/day } \\
\hline 0 & 1.00 & 1.00 & 1.00 & 1.00 \\
\hline$>0-<1$ & $0.47(0.28-0.78)$ & $0.57(0.31-1.06)$ & $0.54(0.34-0.88)$ & $0.66(0.36-1.24)$ \\
\hline $1-4$ & $0.22(0.13-0.40)$ & $0.75(0.30-1.85)$ & $0.32(0.19-0.56)$ & $0.47(0.20-1.11)$ \\
\hline$>4$ & $0.16(0.07-0.35)$ & $0.30(0.08-1.16)$ & $0.45(0.25-0.80)$ & $0.47(0.17-1.31)$ \\
\hline Trend十 & $\mathrm{P}<0.00$ & $\mathrm{P}=0.06$ & $\mathrm{P}=0.02$ & $\mathrm{P}=0.10$ \\
\hline \multicolumn{5}{|c|}{ Leaning on elbow } \\
\hline $0-2$ hours/day & 1.00 & 1.00 & 1.00 & 1.00 \\
\hline$>2$ hours /day & $1.78(1.02-3.12)$ & $2.16(1.06-4.44)$ & $1.32(0.78-2.22)$ & $1.42(0.80-2.53)$ \\
\hline
\end{tabular}

\section{Results}

Table 1 presents the main results. Study 1 showed a negative exposure-response relation between hours of daily computer use and ulnar neuropathy, and to a minor degree with ulnar neuropathy-like symptoms. Participants who used their computer for 4 hours or more per day had a third of the risk for ulnar neuropathy than those who did not use a computer $(\mathrm{OR}=0.30,95 \%$ CI $0.08-1.16)$. For ulnar neuropathy-like symptoms the odds ratio for 4 hours or more of computer use was $0.47,95 \%$ CI $0.17-1.31$. Leaning on the elbow while working implied a higher risk of ulnar neuropathy (OR 2.16, $95 \%$ CI 1.06-4.44), but not for ulnar neuropathy-like symptoms.

\section{Discussion}

This double case-referent study compared patients with ulnar neuropathy and patients with ulnar neuropathy-like symptoms as distinguished by ENG with two separate referent groups, with emphasis on associations with computer work characteristics. A negative exposure-response relationship was found between hours of daily computer use and ulnar neuropathy and the same tendency was seen for ulnar neuropathy-like symptoms. So far, this is the largest and only epidemiological study focusing on com- -puter work as a risk factor for ulnar neuropathy. The study benefited from a double case-referent design that enabled us to judge if observed associations between ulnar neuropathy and computer work reflected specific effects on the ulnar nerve or nonspecific associations with tingling and numbness in the hand. Our analyses included several potential confounders, and we do not think that important potential confounders were left out. This leaves a specific potential causal effect of leaning on an elbow while working, possibly by direct pressure on the nerve in the elbow region, which has been indicated by other authors [2].

The negative findings on hours of daily computer use and both outcomes are in line with the studies of computer use and carpal tunnel syndrome $[10,11]$, and up to now no studies indicate that computer use could be detrimental for the nerves in the arm or hand. From this study preventive efforts in computer work should be reserved for those leaning on an elbow while working, most often the nondominant hand while using mouse devices.

\section{References}

[1] Caliandro P, La TG, Padua R, Giannini F, Padua L. Treatment for ulnar neuropathy at the elbow. Cochrane Database Syst Rev 2011; 2:CD006839.

[2] Descatha A, Leclerc A, Chastang JF, Roquelaure Y; Study Group on Repetitive Work. Incidence of ulnar 
nerve entrapment at the elbow in repetitive work. Scand J Work Environ Health 30 (2004), 234-240.

[3] Latinovic R, Gulliford MC, Hughes RA. Incidence of common compressive neuropathies in primary care. $\mathrm{J}$ Neurol Neurosurg Psychiatry 77(2006), 263-265.

[4] Mondelli M, Giannini F, Ballerini M, Ginanneschi F, Martrelli E. Incidence of ulnar neuropathy at the elbow in The province of Siena (Italy). J Neurol Sci 234 (2005),5-10.

[5] Ohlsson K, Attewell RG, Johnsson B, Ahlm A, Skerfving S.An assessment of neck and upper extremity disorders by questionnaire and clinical examination. Ergonomics 37 (1994), 891-897.

[6] Olney RK. Guidelines in electrodiagnostic medicine. Consensus criteria for the diagnosis of partial conduction block. Muscle Nerve Suppl 8 (1999), 225-229.

[7] Roquelaure Y, Ha C, Leclerc A, Touranchet A, Sauteron $\mathrm{M}$, Melchior $\mathrm{M}$ et al. Epidemiologic surveillance of upper-extremity musculoskeletal disorders in the working population. Arthritis Rheum 55 (2006), 765-778.

[8] Sluiter JK, Rest KM, Frings-Dresen MH. Criteria document for evaluating the work-relatedness of upperextremity musculoskeletal disorders. Scand J Work Environ Health 27 (2001),1-102.

[9] Teschke K, Olshan AF, Daniels JL, De Roos AJ, Parks $\mathrm{CG}$, Schulz $\mathrm{M}$ et al. Occupational exposure assessment in case-control studies: opportunities for improvement. Occup Environ Med 59 (2002), 575-593.

[10] Thomsen JF, Gerr F, Atroshi I (2008) Carpal tunnel syndrome and the use of computer mouse and keyboard: a systematic review. BMC Musculoskeletal Disorders 9 (2008), 134.

[11] Van Rijn RM, Huisstede BM, Koes BW, Burdorf A (2009) Associations between work-related factors and the carpal tunnel syndrome--a systematic review. Scand J Work Environ Health 35 (2009), 19-36. 